\title{
Detection of Pseudomonas aeruginosa in sputum headspace through volatile organic compound analysis
}

Pieter C Goeminne ${ }^{1,4^{*}}$, Thomas Vandendriessche ${ }^{2}$, Johan Van Eldere ${ }^{3}$, Bart M Nicolai ${ }^{2}$, Maarten LATM Hertog ${ }^{2}$ and Lieven J Dupont ${ }^{1}$

\begin{abstract}
Introduction: Chronic pulmonary infection is the hallmark of Cystic Fibrosis lung disease. Searching for faster and easier screening may lead to faster diagnosis and treatment of Pseudomonas aeruginosa (P. aeruginosa). Our aim was to analyze and build a model to predict the presence of $P$. aeruginosa in sputa.

Methods: Sputa from 28 bronchiectatic patients were used for bacterial culturing and analysis of volatile compounds by gas chromatography-mass spectrometry. Data analysis and model building were done by Partial Least Squares Regression Discriminant analysis (PLS-DA). Two analysis were performed: one comparing $P$. aeruginosa positive with negative cultures at study visit (PA model) and one comparing chronic colonization according to the Leeds criteria with $P$. aeruginosa negative patients (PACC model).

Results: The PA model prediction of $P$. aeruginosa presence was rather poor, with a high number of false positives and false negatives. On the other hand, the PACC model was stable and explained chronic $P$. aeruginosa presence for $95 \%$ with 4 PLS-DA factors, with a sensitivity of $100 \%$, a positive predictive value of $86 \%$ and a negative predictive value of $100 \%$.
\end{abstract}

Conclusion: Our study shows the potential for building a prediction model for the presence of chronic $P$. aeruginosa based on volatiles from sputum.

Keywords: Bronchiectasis, Chronic colonization, Gas chromatography mass spectrometry, Cystic fibrosis, Non-cystic fibrosis

\section{Introduction}

Chronic pulmonary infection is the hallmark of Cystic Fibrosis (CF) lung disease. Preventing or treating chronic infection plays a key role in these patients. Previous studies showed that Pseudomonas aeruginosa (P. aeruginosa) infection is associated with lower forced expiratory volume in one second $\left(\mathrm{FEV}_{1}\right)$ during childhood, faster decline in $\mathrm{FEV}_{1}$ during childhood and reduced survival [1-9]. Chronic P. aeruginosa infection is normally preceded by an intermittent presence of the bacteria [10]. Early eradication during this period is important to delay

\footnotetext{
* Correspondence: pieter.goeminne@student.kuleuven.be

'Department of Lung Disease, UZ Leuven, Leuven, Belgium

${ }^{4}$ Pulmonary Medicine, University Hospital Gasthuisberg, Herestraat 49, Leuven B-3000, Belgium

Full list of author information is available at the end of the article
}

chronic colonization [11]. To accomplish early eradication, regular surveillance cultures of sputum are indicated. For non-expectorating patients, oropharyngeal swabs or bronchoalveolar lavage can be used [10].

One of the difficulties measuring successful eradication is proving that the bacteria are completely eliminated from the patient, rather than just temporarily suppressed to a low level that is not detectable, particularly by cough swab $[12,13]$. Sputum culture can be false negative due to overgrowth of other bacteria or (maintenance) treatment with inhaled or oral antibiotics $[14,15]$. A positive culture should not be regarded as a gold standard for diagnosing (chronic) P. aeruginosa infection in CF patients with bronchiectasis and repeated culturing is still a cornerstone of a possible classification based on both bacterial cultures and specific antibody

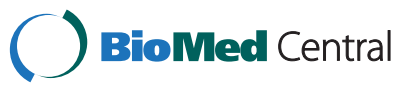


analysis [16]. Repeated culturing is also the cornerstone in non-CF bronchiectasis for the diagnosis of chronic $P$. aerugiosa although different definitions are used [17]. Therefore, other techniques aiming at diagnosis and follow-up of bacterial infection are being investigated. One approach is detection of volatile organic compounds (VOCs) produced by bacteria. $P$. aeruginosa may be detected by analyzing VOCs produced in vitro (Table 1), although the many studies addressing this question measured a variable range of VOCs. Breath or

Table 1 Literature overview of volatile organic compounds found in in vitro and in vivo studies in samples with Pseudomonas aeruginosa

\begin{tabular}{|c|c|c|}
\hline In vitro & Volatiles & reference \\
\hline & $\begin{array}{l}\text { acetaldehyde, acetic acid, acetone, } \\
\text { ammonia, ethanol, dihydrogen sulfide, } \\
\text { dimethyl disulfide, dimethyl sulfide, } \\
\text { methyl mercaptan }\end{array}$ & [21] \\
\hline & $\begin{array}{l}\text { ammonia, hydrogen cyanide, } \\
\text { methyl mercaptan }\end{array}$ & [22] \\
\hline & hydrogen cyanide & [23] \\
\hline & $\begin{array}{l}\text { 2-aminoacetophenone, ammonia, } \\
\text { ethanol, formaldehyde, hydrogen sulfide, } \\
\text { isoprene, methyl mercaptan, } \\
\text { trimethylamine }\end{array}$ & {$[24]$} \\
\hline & $\begin{array}{l}\text { 2-aminoacetophenone, 2-pentanone, } \\
\text { 4-methylphenol, acetic acid, acetone, } \\
\text { acetonitrile, ethanol, ethylene glycol, } \\
\text { indole }\end{array}$ & {$[25]$} \\
\hline & $\begin{array}{l}\text { 1-butanol, 1-undecene, 2-butanone, } \\
\text { 2-heptanone, 2-nonanone, 2-undecanone, } \\
\text { 3-methyl-1-butanol, toluene }\end{array}$ & {$[26]$} \\
\hline & 2-aminoacetophenone & {$[27]$} \\
\hline & $\begin{array}{l}\text { 1-undecene, 2-aminoacetophenone, } \\
\text { 2-butanone, 2-nonanone, 2-undecanone, } \\
\text { 3-methyl-1-butanol, 4-methyl-quinazoline, } \\
\text { butanol, dimethyl disulfide, dimethyl trisulfide, } \\
\text { methyl mercaptan, toluene }\end{array}$ & {$[28]$} \\
\hline & 2-propanol & {$[29]$} \\
\hline & $\begin{array}{l}\text { 2-aminoacetophenone, dimethyl disulfide, } \\
\text { dimethylpyrazine, dimethyl sulfide, } \\
\text { undecene }\end{array}$ & {$[30]$} \\
\hline & Methyl thiocyanate & {$[20]$} \\
\hline \multicolumn{3}{|l|}{ In vivo } \\
\hline breath & hydrogen cyanide & {$[18]$} \\
\hline breath & Methyl thiocyanate & {$[20]$} \\
\hline breath & 2-propanol & [29] \\
\hline sinus mucus & $\begin{array}{l}\text { 2-aminoacetophenone, 2-methylbutyric acid, } \\
\text { 3-hydroxy-2-butanone, acetamide, } \\
\text { acetic acid, acetone, dimethyl disulfide, } \\
\text { dimethyl sulfide, dimethylsulfone, } \\
\text { hydrogen sulfide, indole, isovaleric, } \\
\text { phenol, propanoic acid }\end{array}$ & {$[30]$} \\
\hline sputa & $\begin{array}{l}\text { 1-heptene, 2-nonanone, 2,4-dimethyl-heptene, } \\
\text { 3-methyl-1-butanol, limonene }\end{array}$ & {$[31]$} \\
\hline
\end{tabular}

sputum samples are more challenging to investigate as many factors might influence the VOCs spectrum (eg. recent meal, other bacteria, concomitant medication). A few studies investigating in vivo samples (breath, sinus mucus and sputum) (Table 1 ) suggest that $P$. aeruginosa can be detected via the breath using not only hydrogen cyanide as a single marker [18], but also other biomarkers $[19,20]$. These in vivo studies use bacterial cultures as a gold standard to assess $P$. aeruginosa presence in the sample, not taking into account chronically colonized patients with a false negative sputum culture.

The aims of our study were to predict sputum culture positivity for $P$. aeruginosa in patients with bronchiectasis (PA model) and to predict chronic colonization status with $P$. aeruginosa in patients with bronchiectasis (PACC model) by analysis of the presence of VOCs (Figure 1).

\section{Materials and methods}

\section{Patients}

Consecutive patients who were visiting the outpatient clinic with $\mathrm{CF}$ and non-CF bronchiectasis were included in the study. They were asked to collect their morning sputa, after rinsing their mouth with water and before breakfast, and to bring it to the outpatient clinic. A part of the sputum was used for routine bacterial culture. The second part was used for VOCs analysis within two hours of outpatient clinic visit. Clinical records were reviewed to assess chronic colonization status according to the Leeds criteria [32]. In brief, chronic colonization is diagnosed when more than $50 \%$ of the months, when samples had been taken, were $P$. aeruginosa culture positive. Informed consent was obtained from all patients. Approval was obtained from the local ethical committee of UZ Leuven, Belgium (B51060 - B32220084152).

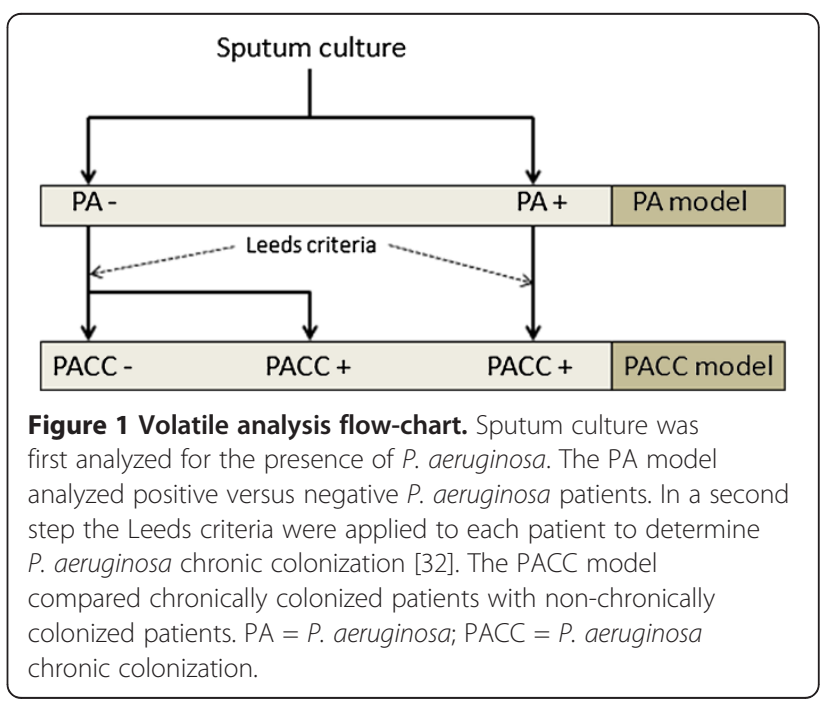




\section{Detection of volatiles}

From every patient 20 grams of morning sputum was transferred into a $10 \mathrm{~mL}$ glass headspace vial (Filter Service, Belgium) within 4 hours from collection, flushed with nitrogen gas and sealed using crimp-top caps with TFE/silicone septa seals (Filter Service, Belgium). Prior to solid phase micro extraction (SPME), the sputum samples were incubated for $24 \mathrm{~h}$ at $37^{\circ} \mathrm{C}$ in a heated tray oven. Headspace volatiles were extracted by exposing a divinylbenzene-carboxen-polydimethylsiloxane SPME fiber (DVB-CAR-PDMS, 50/30 $\mu \mathrm{m}$ film thickness; Supelco Inc., Bellefonte, PA, USA) to the vial headspace for $60 \mathrm{~min}$ at $37^{\circ} \mathrm{C}$. The headspace in our samples is defined by the gaseous constituents of the closed space above the sputum. Every 100 measurements, a new fiber was used. Each fiber was conditioned according to manufacturer's description.

The determination of the VOCs was performed on an Agilent $6890 \mathrm{~N}$ gas chromatograph (GC) (Agilent Technologies, Santa Clara, USA) coupled to an Agilent 5973 Network Mass Selective Detector (MS) (Agilent Technologies, Santa Clara, USA). Automated headspace SPME extraction was performed with an MPS-2 robotic arm (MPS2, Gerstel Multipurpose Sampler, Mülheim an der Ruhr, Germany). After extraction, the VOCs were thermally desorbed into a split/splitless injector heated at $250^{\circ} \mathrm{C}$ and equipped with a SPME liner (0.75 i.d., Supelco Inc., USA). To detect low concentration volatiles, splitless injection was used. Splitless injection was performed for $0.5 \mathrm{~min}$ at $75 \mathrm{~mL} / \mathrm{min}$ and the fiber was further exposed in the injector for $5 \mathrm{~min}$ for thermal conditioning.

Separation was done on an Optima-5-MS capillary column $\left(30 \mathrm{~m} \times 0.25 \mathrm{~mm}\right.$ i.d. $\times 0.25 \mu \mathrm{m} \mathrm{d}_{\mathrm{f}}$ ) (MachereyNagel, Germany). Helium was used as carrier gas under a constant flow of $1.0 \mathrm{~mL} / \mathrm{min}$. The GC temperature program started isothermal at $35^{\circ} \mathrm{C}$ for $3 \mathrm{~min}$ and was then ramped to $250^{\circ} \mathrm{C}$ at a rate of $10^{\circ} \mathrm{C} \mathrm{min}{ }^{-1}$. Finally, the temperature was kept isothermal at $250^{\circ} \mathrm{C}$ for $5 \mathrm{~min}$. The total run time was $29.50 \mathrm{~min}$. The GC interface temperature was $280^{\circ} \mathrm{C}$.

Mass spectra in the 15 to $350 \mathrm{~m} / \mathrm{z}$ range were recorded at a scanning speed of 4.15 scans cycles per second. The MS source and quadrupole temperatures were $230^{\circ} \mathrm{C}$ and $150^{\circ} \mathrm{C}$ respectively. The chromatography and spectral data were evaluated using the MSD ChemStation Software (Agilent Technologies, Santa Clara, USA) and AMDIS v. 2.1 (Automated Mass Spectral Deconvolution and Identification System, NIST, Gaithersburg, MD, USA). Only those compounds with a signal to noise ratio $>20$ and that could be identified through comparison with the spectral library NIST having a match and reversed match percentage $>80 \%$ and from which additionally the spectrum was manually controlled, were included in the analysis. The volatile compounds were identified by comparing the experimental spectra with those of the National Institute for Standards and Technology (NIST98 v. 2.0, Gaithersburg, $\mathrm{MD}, \mathrm{USA}$ ) and by retention indices. The retention time is the characteristic time it takes for a specific volatile to pass through the system. The (Kovats) retention index of a compound is its retention time normalized to the retention times of adjacently eluting $\mathrm{n}$-alkanes. They help to identify components by comparing experimentally found retention indices with known values. The Kovats retention index is used to allow other analytical laboratories to compare measured values. We evaluated VOCs with a molecular weight higher than 30. Lower molecular weight VOCs (such as Hydrogen Cyanide) could not be evaluated as too many small compounds were co-eluting in the beginning of the chromatogram. Therefore, it was not possible to determine their presence in a reliable way (even with deconvolution programs). Hydrocarbon standards $\left(\mathrm{C}_{8}\right.$ to $\mathrm{C}_{20}$ in hexane, Sigma-Aldrich, Steinheim, Germany) were injected using the same GC-MS method to determine the retention indices of the individual compounds using a modified Kovats method [33].

\section{Bacterial culturing}

Sputa were inoculated on standard culture media (Blood agar with optochin disc, Mannitol Salt agar and MacConkey agar). Selective culture media were used for Haemophilus spp. (Haemophilus agar) Burkholderia cepacia complex (Mast B. cepacia complex agar) and fungi (Sabouraud agar)

\section{Pseudomonas aeruginosa (PA) model}

For the PA model, we compared patients with a P. aeruginosa positive sputum culture at study to those with a negative $P$. aeruginosa sputum culture at study visit (Figure 1).

\section{Pseudomonas aeruginosa chronically colonized (PACC) model}

For the PACC model, we compared patients with a known $P$. aeruginosa colonization according to the Leeds criteria to those without $P$. aeruginosa colonization at study visit (Figure 1) [32].

\section{Multivariate data analysis}

All data was evaluated using multivariate data analysis techniques, including Principal Component Analysis (PCA) and Partial least-squares discriminant analysis (PLS-DA). The former is an unsupervised explorative method which is based on the principle of latent variables. It transforms large multivariate datasets of correlated variables into a new (reduced) dataset containing 
orthogonal (uncorrelated) variables only, named principal components. The latter is then used to reveal the relation of the samples to a given parameter, where the predictor variable is used in the calculation of the latent variables. The goal is to describe as much of the response variation and to search for directions that are relevant with respect to the predictor variable. The obtained PLS model can be further used to predict the predictor variable response for unknown samples. Data preprocessing steps included mean centering and weighing of all variables by their standard deviation to give them equal variance. In order to evaluate every dataset before analysis, a PCA was conducted to detect possible outlying samples by means of the $95 \%$ Hotelling's $\mathrm{T}^{2}$ limit. Hotelling's T-squared statistic is a generalization of Student's t statistics that is used in multivariate hypothesis testing. Two samples were discarded from the dataset due to technical failure during measurement. PLSDA, a supervised technique, was used to discriminate between non-infected patients versus patients infected with $P$. aeruginosa or chronically colonized patients versus noncolonized patients. In order to test the performance of the models, a segmented $(4 \times 7)$ cross-validation was applied. The quality of the model was evaluated by using the $\mathrm{R}^{2}$-value between measured and predicted. The Variable Identification (VID) coefficients were calculated to identify possible biomarkers. The VID coefficient was calculated as the correlation coefficient between each original $\mathrm{X}$-variable and the $\mathrm{Y}$-variable as predicted by the PLS-DA model [34]. The VID is therefore important to understand what the potential relevance of each aroma compound is with respect to the predictor variable. PCA and PLS-DA analyses were performed using Unscrambler vs 9.8 (CAMO Technologies Inc., Woodbridge, USA).

\section{Results \\ Population}

During the study period 30 patients were recruited and sputum was analyzed of 28 patients (male (43\%); average age 29 y \pm 12 ; $11 \%$ non-CF bronchiectasis and $89 \%$ CF). Two samples were discarded from the dataset due to technical failure during measurement. Bacterial culturing of the 28 patients showed that 14 patients had $P$. aeruginosa in their sputa (50\%) collected at the time of the study. Five patients did not grow $P$. aeruginosa in sputum culture but were known to be chronically colonized according to the Leeds criteria [32]. The remaining nine patients had no history of having $P$. aeruginosa cultured in their sputum. The patients with chronic $P$. aeruginosa colonization had an average IgG for $P$. aeruginosa of $40 \mathrm{AU}$.

All but one patient were taking antibiotics as treatment, either with a single or a combined scheme of antibiotics (68\% on chronic macrolide therapy, $54 \%$ on inhaled tobramycin and/or on inhaled colistimethate; $11 \%$ on oral penicillines; $14 \%$ on oral quinolones; $7 \%$ on oral cefalosporins, $4 \%$ on oral clindamycin and $7 \%$ on oral co-trimoxazoles.) Two of the patients on oral antibiotics took their oral antibiotic treatment as maintenance therapy and the other nine received it due to an exacerbation they had suffered. In addition to $P$. aeruginosa, bacterial culture isolated Staphylococcus aureus in $36 \%$, Aspergillus fumigatus in 29\%, Achromobacter xylosoxidans in $11 \%$, Haemophilus influenza in $7 \%$ and $B$. cepacia complex in $7 \%$.

\section{GC-MS results}

Around one hundred aroma compounds were detected using the deconvolution software AMDIS. This resulted in 61 VOCs (Table 2) of which the retention indexes (RI) were also checked.

\section{Multivariate data analysis PA model}

In the PA model, $P$. aeruginosa positivity was based on sputum culture positivity for $P$. aeruginosa at study visit, excluding the patients known to be chronically colonized from the $P$. aeruginosa positives. The PA model showed an explained variance of $95 \%$ after 9 PLS-DA Factors but showed an unstable validation. It also showed less good prediction for the presence of PA in sputum culture with high number of false positives and false negatives. Sensitivity was $72 \%$, specificity was $40 \%$, positive predicted value was $63 \%$ and negative predicted value was $67 \%$ (Figure 2).

\section{PACC model}

Our PACC model included all $P$. aeruginosa chronically colonized patients, even if sputum culture at study visit was negative. The PACC model can explain the colonization status with $P$. aeruginosa with an explained variance of $95 \%$ with 4 PLS-DA Factors, and a stable validation. It showed a good prediction of presence with $P$. aeruginosa. The PACC model had no false negatives, but there were three false positive (Figure 3). This means our PACC model has a sensitivity of $100 \%$, a specificity of $67 \%$, a positive predictive value of $86 \%$ and a negative predictive value of $100 \%$.

\section{Volatile analysis of the PACC model}

Based on the PLS-DA, the Variable Identification (VID) coefficients were calculated in order to examine the relationship between each VOC and the presence of $P$. aeruginosa. VID coefficients showed a positive and negative correlation with the presence of certain VOCs, although most correlation loadings were low (Table 2). This can also be perceived in the correlation loadings plots 
Table 2 Overview of all volatile organic compounds studied with their respective retention time (RT), Kovats retention index (RI) and variable identification coefficients (VID) in the PACC model

\begin{tabular}{|c|c|c|c|}
\hline Name & RT & KRI & $\overline{\text { VID }}$ \\
\hline 1R-a-pinene & 8,872 & 937,3333 & 0.42 \\
\hline 2,2,6-trimethyl-octane & 9,363 & 963,52 & 0.42 \\
\hline dodecane & 13,29 & 1200 & 0.40 \\
\hline terpinen-4-ol & 13,03 & 1183,14 & 0.40 \\
\hline 1-undecene & 11,6 & 1091,77 & 0.37 \\
\hline 3,7-dimethyl-1,6-octadien-3-ol & 11,73 & 1099,704 & 0.32 \\
\hline 2,6,7-trimethyl- decane & 11,03 & 1058,378 & 0.31 \\
\hline indole & 14,69 & 1296,944 & 0.31 \\
\hline toluene & 5,377 & 759,4782 & 0.31 \\
\hline ethanol & 1,746 & $<600$ & 0.31 \\
\hline 3-hydroxy-2-butanone & 4,261 & 697,5298 & 0.30 \\
\hline acetic acid & 2,673 & 609,3811 & 0.28 \\
\hline amylene hydrate & 3,046 & 630,086 & 0.27 \\
\hline caryophyllene & 16,55 & 1438,148 & 0.26 \\
\hline 1-methyl-4-(1-methylethyl)-cyclohexanol & 12,95 & 1178,121 & 0.26 \\
\hline 2,5-dimethyl-2,5-hexanediol & 8,466 & 915,68 & 0.25 \\
\hline 2-nonanone & 11,56 & 1089,816 & 0.25 \\
\hline acetone & 1,859 & $<600$ & 0.22 \\
\hline 2-ethyl-1-hexanol & 10,54 & 1029,248 & 0.22 \\
\hline 2-heptanone & 7,947 & 889,1041 & 0.21 \\
\hline 2-ethoxy-2-methyl-propane & 2,801 & 616,4863 & 0.21 \\
\hline phenylethyl alcohol & 11,97 & 1115,187 & 0.18 \\
\hline 1-octen-3-ol & 9,66 & 979,36 & 0.18 \\
\hline 4-methyl octane & 7,46 & 865,5206 & 0.17 \\
\hline 1-butanol, 3-methyl-, acetate & 7,686 & 876,4649 & 0.15 \\
\hline 1-butanol, 3-methyl- & 4,796 & 727,2273 & 0.14 \\
\hline d-limonene & 10,6 & 1032,445 & 0.14 \\
\hline Eucalyptol & 10,64 & 1034,991 & 0.14 \\
\hline 6-methyl-2-heptanone & 9,192 & 954,4 & 0.14 \\
\hline Thymol & 14,62 & 1292,222 & 0.12 \\
\hline Benzeneacetaldehyde & 10,76 & 1042,214 & 0.12 \\
\hline 2-hexanone & 5,859 & 786,2337 & 0.11 \\
\hline 2,4-dimethyl-1-heptene & 7,004 & 843,4383 & 0.11 \\
\hline 5-methyl-2-(1-methylethyl)-cyclohexanone & 12,63 & 1157,529 & 0.10 \\
\hline 2,4-dimethyl-heptane & 6,599 & 823,8257 & 0.09 \\
\hline Pyrollidine & 3,583 & 659,8945 & 0.08 \\
\hline 2,3-dimethyl-heptane & 7,3 & 857,7724 & 0.06 \\
\hline 2,6-dimethyl-7-octen-2-ol & 11,27 & 1072,114 & 0.05 \\
\hline 3-methyl-2-pentanone & 5,085 & 743,2695 & -0.03 \\
\hline 2-undecanone & 14,61 & 1291,597 & -0.04 \\
\hline 3-octanone & 9,737 & 983,4667 & -0.08 \\
\hline methyl isobutyl ketone & 4,806 & 727,7824 & -0.08 \\
\hline
\end{tabular}

Table 2 Overview of all volatile organic compounds studied with their respective retention time (RT), Kovats retention index (RI) and variable identification coefficients (VID) in the PACC model (Continued)

\begin{tabular}{lrrr}
\hline phenol & 9,733 & 983,2533 & -0.12 \\
3-methyl-3-buten-1-ol & 4,686 & 721,1213 & -0.14 \\
2-pentyl-furan & 9,89 & 991,6267 & -0.15 \\
3-methyl butanal & 3,194 & 638,3014 & -0.16 \\
1-propanol & 2,213 & $<600$ & -0.19 \\
3-methyl-, (ethyl ester) butanoic acid & 7,213 & 853,5593 & -0.20 \\
octane & 6,122 & 800,7264 & -0.22 \\
1-butanol & 3,487 & 654,5656 & -0.22 \\
2-butanone & 2,492 & $<600$ & -0.23 \\
2-pentanone & 3,776 & 670,6078 & -0.24 \\
thiocyanic acid, methyl ester & 4,188 & 693,4777 & -0.24 \\
2-methyl-,(ethyl ester) butanoic acid & 7,151 & 850,5569 & -0.26 \\
2-methyl butanal & 3,355 & 647,2384 & -0.27 \\
ethyl acetate & 2,709 & 611,3794 & -0.28 \\
hexane & 2,528 & 601,3322 & -0.38 \\
dimethyl tetrasulfide & 13,5 & 1214,583 & -0.43 \\
dimethyl disulfide & 4,878 & 731,7791 & -0.46 \\
dimethyl trisulfide & 9,5 & 970,8267 & -0.47 \\
2-methyl-pentane & 2,259 & $<600$ & -0.59 \\
\hline Volatile Organic Compounds (VOCs) were ranked according their VID with \\
high values indicating a positive correlation with Pseudomonas aeruginosa \\
infection and negative values indicating a negative correlation; KRI = Kovats \\
Retention Index. & & \\
\end{tabular}

(Figure 4). Using two principle compounds, $86 \%$ of $P$. aeruginosa presence can be explained through the PACC model. There's a clear separation between $P$. aeruginosa positive and negative patients in the correlation loadings plot (Figure 4). VOCs analysis shows that the five largest negative correlations can be seen for the sulphur compounds dimethyl disulfide (VID $=-0.46)$, dimethyl trisulfide (VID $=-0.47$ ) and dimethyl tetrasulfide (VID = $-0.43)$ and two other compounds: hexane (VID $=-0.38$ ) and 2-methyl pentane (VID $=-0.59$ ). The five largest positive correlations were found for the terpenes 1undecene (VID $=0.37)$ and $1-\alpha$-pinene $(\mathrm{VID}=0.42)$ and the compounds dodecane (VID $=0.40$ ), terpinen-4-ol $(\mathrm{VID}=0.40)$ and 2,2,6-trimethyl-octane $(\mathrm{VID}=0.42)$ (Table 2).

Exclusion of the non-CF bronchiectatic patients from the PLS analysis, analyzing only the CF population did not change the results in terms of positions of the VOCs and amount of $\mathrm{X}(=\mathrm{VOCs})$ and $\mathrm{Y}(=P$. aeruginosa $)$ variation explained (data not shown).

\section{Discussion}

Our study shows that it may be possible to use the presence of VOCs in sputum to assess the presence of $P$. 


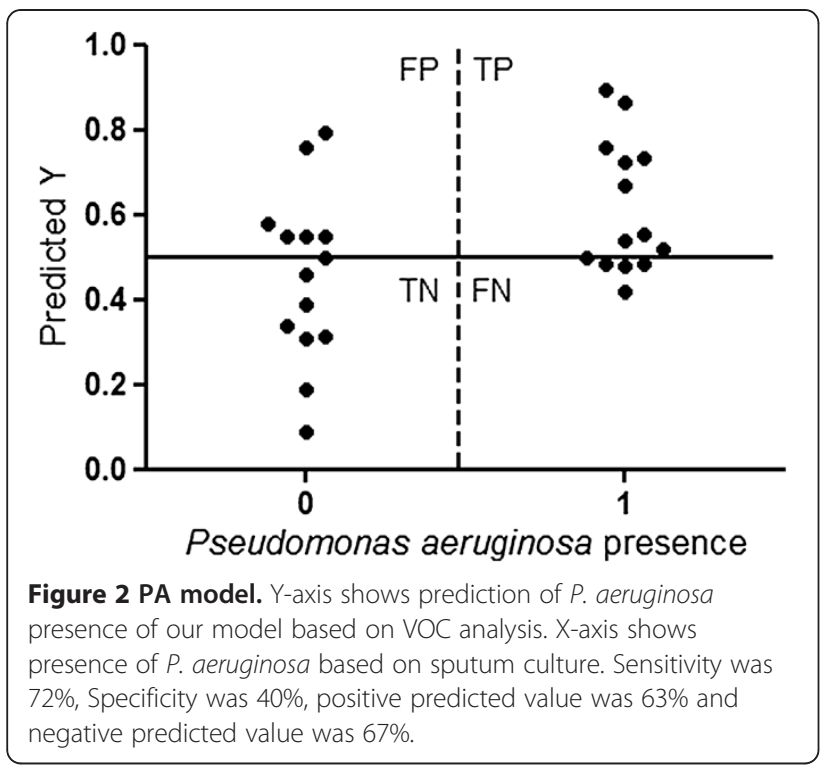

aeruginosa and colonization status with $P$. aeruginosa. Analysis showed that not a single but a pattern of VOCs are linked to the presence of $P$. aeruginosa. VOCs that were positively associated with $P$. aeruginosa included the terpenes 1-undecene, 1- $\alpha$-pinene, dodecane, terpinen-4-ol and 2,2,6-trimethyl-octane. A more pronounced negative correlation can be seen for the sulphur compounds dimethyl disulfide, dimethyl trisulfide and dimethyl tetrasulfide with the addition of hexane and 2-methyl-pentane. The results of the PACC model showed a sensitivity and negative predictive value of $100 \%$. This suggests that, based on VOCs analysis, our model is able to predict chronic colonization with

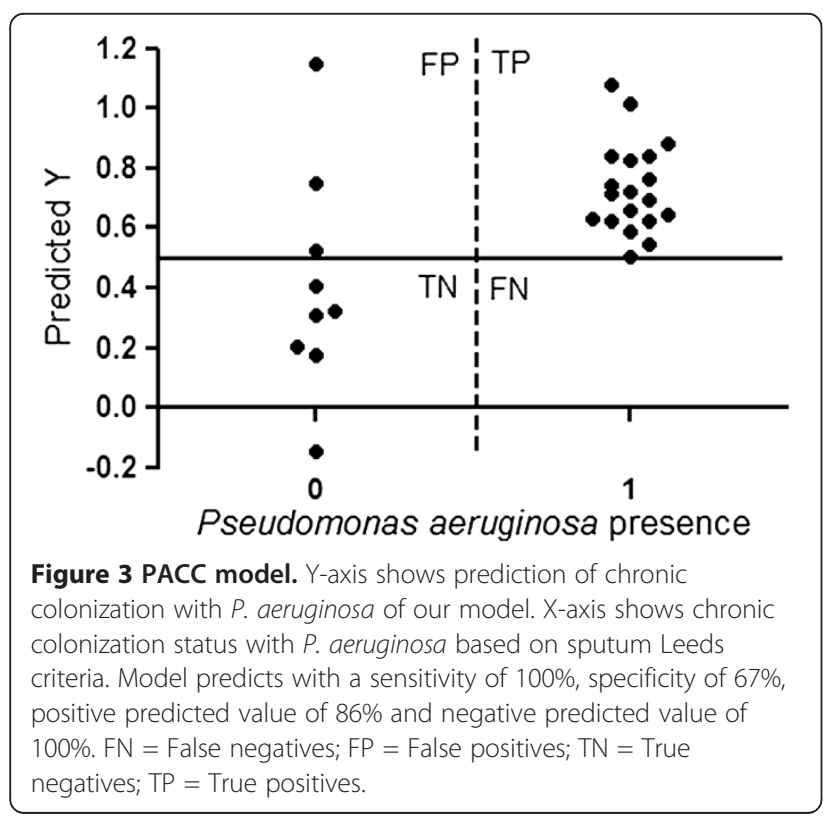

$P$. aeruginosa. Some of the patients known with chronic colonization of $P$. aeruginosa had a negative sputum culture for $P$. aeruginosa at study visit. This suggests that gas chromatography - mass spectrometry may be more sensitive than bacterial culturing.

Previous studies have shown that several VOCs in sputa, breath and mucus may indicate the presence of $P$. aeruginosa [18,29-31]. Our study results confirm that most of these VOCs were present in sputum from patients with $P$. aeruginosa, but none of these VOCs were highly specific for the presence of $P$. aeruginosa. We could not identify one single VOC that was representative for the presence of $P$. aeruginosa presence.

In our study, the presence and absence of a library of 61 VOCs was identified and found to discriminate between patients with and without $P$. aeruginosa in sputum. Some of the VOCs we identified in the sputum headspace samples were the same as those found in other studies. If we compare the results with the study of Savelev et al. we can find their suggested markers in our samples [31]. They looked for specific biomarkers, showing the highest individual sensitivity for 2-nonanone. Although our specific aim was to look for a prediction model, rather than searching and evaluating individual candidate biomarkers, we found a similar positive correlation with 2-nonanone ( $\mathrm{VID}=0.25)$, limonene $(\mathrm{VID}=0.14), 2,4$-dimethyl-heptene $(\mathrm{VID}=0.11)$ and 3-methyl-1-butanol (VID=0.14).

A clear distinction needs to be made between VOCs analysis of bacterial cultures (in vitro studies) and patient in vivo sample analysis. One typical example is 2-aminoacetophenone. 2-aminoacetophenone is known for its sweet grape-like odour. On culture plates growing $P$. aeruginosa $[27,28]$, its odour increases when adding tryptophan. This is because 2-aminoacetophenone is an intermediate in the biosynthetic pathway for quinazolines, a pathway branching from the tryptophan catabolic pathway. Conversely, only one in vivo study could show its presence in trace quantities [30]. This indicates that the VOCs profile produced by $P$. aeruginosa in vivo may differ from its in vitro VOCs production and cannot be extrapolated from in vitro to in vivo analysis purposes, as culture media can have an impact on VOCs. Moreover, most in vitro studies are explorative studies, describing the spectrum of VOCs in different bacterial cultures without assessing them as biomarkers (such as dimethyl disulfide and dimethyl sulfide), with the exception of hydrogen cyanide [21,23], 2-propanol [29] and methyl thiocyanate [20]. Hydrogen cyanide, 2-propanol and methyl thiocyanate were also found in in vivo samples (breath). Hydrogen cyanide was not evaluated as our GC-MS results only allowed reliable evaluation of VOCs with a molecular weight higher than 30 . For 2propanol, the isomer 1-propanol could be detected but 


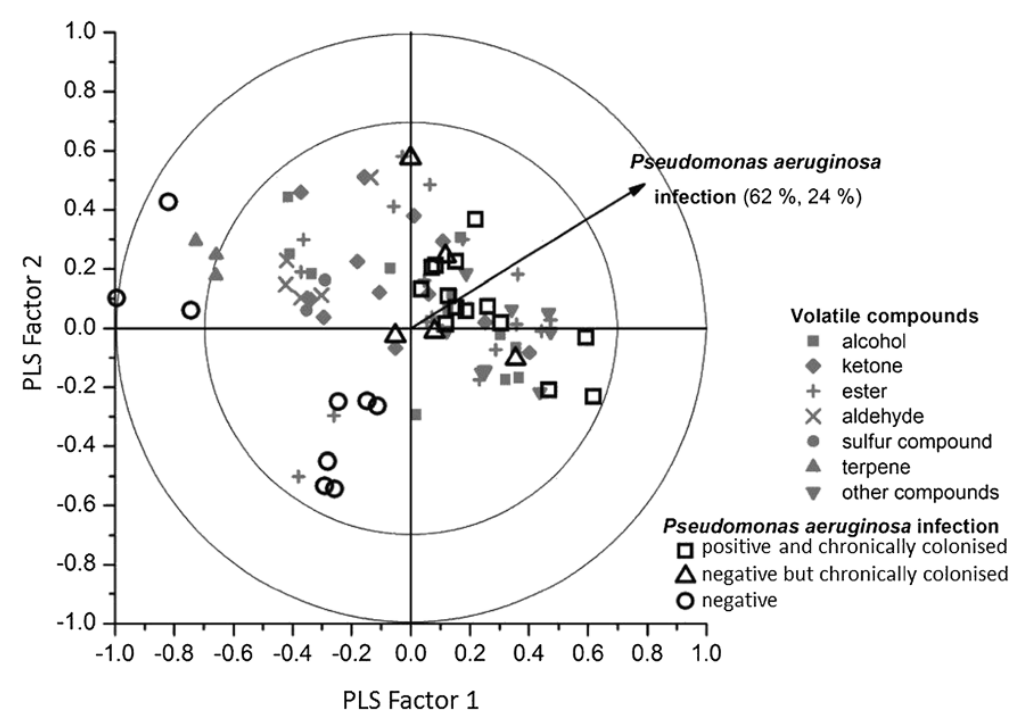

Figure 4 Biplot using the first two PLS-DA factors. The plot shows a good separation of $P$. aeruginosa positive chronic colonized patients (triangles and squares) and $P$. aeruginosa negative patients (circles). Significant correlation of volatiles is suggested when volatiles project between $r=0.75$ (inner circle) and $r=1$ (outer circle). The vector shows the direction where volatiles are positively correlated with chronic $P$. aeruginosa. The pattern of volatiles could explain P. aeruginosa infection in 86\% using the first two PLS-DA factors (62\% and 24\%). X and $Y$ axis both show partial least square regression r. Each PLS factor explains $10 \%$ and $6 \%$ of the X-variation respectively. The light gray symbols visualize the volatile organic compounds, sorted by structure. Squares: Chronic colonization and positive sputum cultures for $P$. aeruginosa at the time of study. Triangles: Chronic colonization but negative sputum culture for $P$. aeruginosa at the time of study. Circles: Negative for $P$. aeruginosa.

was also seen in samples without $P$. aeruginosa. Methyl thiocyanate (or thiocyanic acid, methyl ester) was not associated with $P$. aeruginosa in our samples. Shestivska et al. could not find methyl thiocyanate in some P. aeruginosa strains. This means that methyl thiocyanate is strain specific and might explain its absence in our study population.

The different results on the presence of VOCs shown in some previous studies (Table 1) raises the question if not a single VOC is indicative of $P$. aeruginosa presence but rather a pattern of VOCs, as suggested by our results. However we did not analyze VOCs with a molecular weight lower than 30. Recently, strong evidence showed that hydrogen cyanide could be used as a biomarker, showing significant higher in vivo concentrations in most strains of $P$. aeruginosa [18]. This biomarker could then be used in the detection of $P$. aeruginosa in breath, whether or not in combination with $\mathrm{CH}_{3} \mathrm{SCN}$ (methyl thiocyanate) as possible biomarker [20]. Further research is warranted to identify a single biomarker or a pattern of VOCs ("a breathogram"). This would mean the addition of a new tool for the diagnosis of (chronic) P. aeruginosa infection and the monitoring of response to treatment (eg eradication therapy) [35].

The use of novel devices using the breath end portion of a normal spirometry measurement to perform a chromatographic preseparation, followed by an ion mobility spectrometry (IMS) or devices allowing fast analysis of breath using a selected ion flow tube mass spectrometry
(SIFT-MS) make it fast and feasible to do VOCs analysis [36,37]. SIFT-MS has the advantage of being fast and having high sensitivity. It can also determine the endtidal breath phase by quantification of water vapour in breath samples while the soft ionization technique allows easy analysis of high moisture samples such as breath. IMS has the disadvantage of not knowing what chemical compound is seen unless a large database with standards is available, but it has been proven that IMS is also fast and can show a fingerprint, characteristic for an infection [38].

A limitation of our study might be the impact other variables have on VOCs such as antibiotic therapy and other bacteria. Bacterial culture results from all our patients showed a great diversity and variability without a distinct pattern of bacterial co-existence between patients. More importantly, our statistical design, using PLS-DS, minimizes the impact of variables such as antibiotic therapy and other bacteria. PLS-DS reveals the relation of the samples to a given parameter, particularly $P$. aeruginosa.

Our findings of terpenes and terpenoids in sputum headspace are interesting as they are common constituents of food. Alpha-pinene for example is detected in fruits and pepper. Although we asked the patients to produce their sputa after rinsing their mouth and before breakfast, we cannot reliably say this was done by the patient. However, if the detected VOCs would indeed be related to food, this would mean that all patients with 
P. aeruginosa had the same food VOCS constituents in their breath.

Quantification of the VOCs was also not performed. To perform quantification for complex matrices, the use of internal standards or standard additions is recommended. Using only a few internal standards, representing the main chemical classes and extrapolating the results to all volatiles in the sample, can cause serious errors. Ideally SPME quantification would require us to focus on a few volatiles (which was not our aim) and use isotopically labeled analogues as standards. Although we did not quantify, all samples were processed and analyzed in a same manner, reducing the variability due to the methods. This results in a variability mainly due to the sample itself.

Another important issue that should be taken into consideration is that sputum might be contaminated by saliva, influencing the results of the VOC analysis. This has been proven for breath analysis, where important contamination of alveolar breath exhaled via the mouth can occur [39]. Wang et al. showed that both mouthand nose-exhaled breath analyses are needed to identify the major source of a certain VOC. We tried to minimize the effect of saliva contamination by asking the patient to rinse their mouth prior to sputum production. Nonetheless, finding a biomarker for $P$. aeruginosa in mouth VOCs would still be interesting as current literature suggests that a migration from $P$. aeruginosa is seen from the upper to the lower airways prior to colonization [40].

\section{Conclusion}

We showed that building a model for the prediction of $P$. aeruginosa presence is possible and might even identify known chronic colonized patients as $P$. aeruginosa where sputum culture cannot show its presence. Based on literature overview and our results, we believe that not the presence of a single VOC is indicative of $P$. aeruginosa presence but rather a pattern of VOCs. Follow-up of patients, producing a "breathogram" might be a promising future perspective, but needs further research, using new devices such as spirometry combined with chromatographic preseparation and subsequent ion mobility spectrometry.

\footnotetext{
Abbreviations

CF: Cystic fibrosis; FEV 1 : Forced expiratory volume in one second; GC-MS: Gas chromatography - mass spectrometry; IMS: Ion mobility spectrometry; $P$. aeruginosa: Pseudomonas aeruginosa; PACC: Pseudomonas aeruginosa chronic colonization; PCA: Principal component analysis; PLS-DA: Partial least square discriminant analysis; RI: Retention index; RT: Retention time; SIFTMS: Selected ion flow tube mass spectrometry; SPME: Solid phase micro extraction; VID: Variable identification; VOCs: Volatile organic compounds.
}

\section{Competing interest}

None of the authors has a financial relationship with a commercial entity that has an interest in the subject of the presented manuscript.

\section{Authors' contribution}

PG performed the acquisition and analysis of the data, designed the study and wrote the manuscript. TV aided in the data acquisition and data processing, performed part of the analysis and reviewed the article. JVE was involved in the design of the study and reviewed the article. $\mathrm{MH}$ contributed importantly to the interpretation of the data and critically revised the manuscript. BN was involved in the design of the study and critical revision of the manuscript. LD was involved in the design and critical revision prior to submission. All authors read and approved the final manuscript.

\section{Acknowledgements}

We would like to thank Elfie Dekempeneer for her advice and help during the measurements of the samples. We also thank Rita Merckx for her help and advice concerning bacterial culturing and we thank Stijn Willems for his critical review of the manuscript.

\section{Author details}

${ }^{1}$ Department of Lung Disease, UZ Leuven, Leuven, Belgium. ²Biosyst-MeBios, University of Leuven, Leuven, Belgium. ${ }^{3}$ Department of Microbiology, UZ Leuven, Leuven, Belgium. ${ }^{4}$ Pulmonary Medicine, University Hospital Gasthuisberg, Herestraat 49, Leuven B-3000, Belgium.

Received: 18 July 2012 Accepted: 27 September 2012

Published: 2 October 2012

\section{Reference}

1. Konstan MW, Morgan WJ, Butler SM, Pasta DJ, Craib ML, Silva SJ, Stokes DC, Wohl ME, Wagener JS, Regelmann WE, Johnson CA: Scientific advisory group and the investigators and coordinators of the epidemiologic study of cystic fibrosis. Risk factors for rate of decline in forced expiratory volume in one second in children and adolescents with cystic fibrosis. J Pediatr 2007, 151:134-139.

2. McPhail GL, Acton JD, Fenchel MC, Amin RS, Seid M: Improvements in lung function outcomes in children with cystic fibrosis are associated with better nutrition, fewer chronic pseudomonas aeruginosa infections, and dornase alfa use. J Pediatr 2008, 153:752-757.

3. Kerem E, Corey M, Gold R, Levison H: Pulmonary function and clinical course in patients with cystic fibrosis after pulmonary colonization with Pseudomonas aeruginosa. J Pediatr 1990, 116:714-719.

4. Emerson J, Rosenfeld M, McNamara S, Ramsey B, Gibson RL: Pseudomonas aeruginosa and other predictors of mortality and morbidity in young children with cystic fibrosis. Pediatr Pulmonol 2002, 34:91-100.

5. Kozlowska WJ, Bush A, Wade A, Aurora P, Carr SB, Castle RA, Hoo AF, Lum S, Price J, Ranganathan S, Saunders C, Stanojevic S, Stroobant J, Wallis C, Stocks J: London cystic fibrosis collaboration. Lung function from infancy to the preschool years after clinical diagnosis of cystic fibrosis. Am J Respir Crit Care Med 2008, 178:42-49.

6. Pamukcu A, Bush A, Buchdahl R: Effects of pseudomonas aeruginosa colonization on lung function and anthropometric variables in children with cystic fibrosis. Pediatr Pulmonol 1995, 19:10-15.

7. Kosorok MR, Zeng L, West SE, Rock MJ, Splaingard ML, Laxova A, Green CG Collins J, Farrell PM: Acceleration of lung disease in children with cystic fibrosis after pseudomonas aeruginosa acquisition. Pediatr Pulmonol 2001, 32:277-287.

8. Henry RL, Mellis CM, Petrovic L: Mucoid pseudomonas aeruginosa is a marker of poor survival in cystic fibrosis. Pediatr Pulmonol 1992, 12:158-161.

9. Courtney JM, Bradley J, Mccaughan J, O'Connor TM, Shortt C, Bredin CP, Bradbury I, Elborn JS: Predictors of mortality in adults with cystic fibrosis. Pediatr Pulmonol 2007, 42:525-532.

10. Burns JL, Gibson RL, McNamara S, Yim D, Emerson J, Rosenfeld M, Hiatt P, McCoy K, Castile R, Smith AL, Ramsey BW: Longitudinal assessment of pseudomonas aeruginosa in young children with cystic fibrosis. $J$ Infect Dis 2001, 183:444-452

11. Hansen CR, Pressler T, Hoiby N: Early aggressive eradication therapy for intermittent pseudomonas aeruginosa airway colonization in cystic fibrosis patients: 15 years experience. J Cyst Fibros 2008, 7:523-530.

12. Armstrong DS, Grimwood K, Carlin JB, Carzino R, Olinsky A, Phelan PD: Bronchoalveolar lavage or oropharyngeal cultures to identify lower respiratory pathogens in infants with cystic fibrosis. Pediatr Pulmonol 1996, 21:267-275. 
13. Endeman $H$, Schelfhout V, Voorn GP, van Velzen-Blad H, Grutters JC, Biesma DH: Clinical features predicting failure of pathogen identification in patients with community acquired pneumonia. Scand J Infect Dis 2008, 40:715-720.

14. Terpstra WJ, Schoone GJ, Ter SJ, van Nierop JC, Griffioen RW: In situ hybridization for the detection of haemophilus in sputum of patients with cystic fibrosis. Scand J Infect Dis 1987, 19:641-646.

15. Verenkar MP, Pinto MJ, Savio R, Virginkar N, Singh I: Bacterial pneumoniasevaluation of various sputum culture methods. J Postgrad Med 1993, 39:60-62.

16. Pressler T, Bohmova C, Conway S, Dumcius S, Hjelte L, Høiby N, Kollberg H, Tümmler B, Vavrova V: Chronic pseudomonas aeruginosa infection definition: EuroCareCF working group report. J Cyst Fibros 2011, 10:575-S78.

17. Pasteur MC, Bilton D, Hill AT: British Thoracic Society guideline for non-CF bronchiectasis. Thorax 2010, 65:i1-i58.

18. Enderby B, Smith D, Carroll W, Lenney W: Hydrogen cyanide as a biomarker for Pseudomonas aeruginosa in the breath of children with cystic fibrosis. Pediatr Pulmonol 2009, 44:142-147.

19. Robroeks CM, van Berkel JJ, Dallinga JW, Jöbsis Q, Zimmerman LJ, Hendriks HJ, Wouters MF, van der Grinten CP, van de Kant KD, van Schooten FJ, Dompeling E: Metabolomics of volatile organic compounds in cystic fibrosis patients and controls. Pediatr Res 2010, 68:75-80.

20. Shestivska V, Nemec A, Dřevínek P, Sovová K, Dryahina K, Spaněl P: Quantification of methyl thiocyanate in the headspace of pseudomonas aeruginosa cultures and in the breath of cystic fibrosis patients by selected ion flow tube mass spectrometry. Rapid Commun Mass Spectrom 2011, 25:2459-2467.

21. Allardyce RA, Langford VS, Hill AL, Murdoch DR: Detection of volatile metabolites produced by bacterial growth in blood culture media by selected ion flow tube mass spectrometry (SIFT-MS). J Microbiol Methods 2006, 65:361-365.

22. Carroll W, Lenney W, Wang T, Spanel P, Alcock A, Smith D: Detection of volatile compounds emitted by pseudomonas aeruginosa using selected ion flow tube mass spectrometry. Pediatr Pulmonol 2005, 39:452-456.

23. Gilchrist FJ, Alcock A, Belcher J, Brady M, Jones A, Smith D, Spaněl $P$, Webb K, Lenney W: Variation in hydrogen cyanide production between different strains of pseudomonas aeruginosa. Eur Respir J 2011, 38:409-414

24. Thorn RM, Reynolds DM, Greenman J: Multivariate analysis of bacterial volatile compound profiles for discrimination between selected species and strains in vitro. J Microbiol Methods 2011, 84:258-264.

25. Zhu J, Bean HD, Kuo YM, Hill JE: Fast detection of volatile organic compounds from bacterial cultures by secondary electrospray ionization-mass spectrometry. J Clin Microbiol 2010, 48:4426-4431.

26. Zechman JM, Labows JN Jr: Volatiles of Pseudomonas aeruginosa and related species by automated headspace concentration-gas chromatography. Can J Microbiol 1985, 31:232-237.

27. Cox CD, Parker J: Use of 2-aminoacetophenone production in identification of pseudomonas aeruginosa. J Clin Microbiol 1979, 9:479-484.

28. Labows JN, McGinley KJ, Webster GF, Leyden JJ: Headspace analysis of volatile metabolites of pseudomonas aeruginosa and related species by gas chromatography-mass spectrometry. J Clin Microbiol 1980, 12:521-526.

29. Wang T, Carroll W, Lenny W, Boit P, Smith D: The analysis of 1-propanol and 2-propanol in humid air samples using selected ion flow tube mass spectrometry. Rapid Commun Mass Spectrom 2006, 20:125-130.

30. Preti G, Thaler E, Hanson CW, Troy M, Eades J, Gelperin A: Volatile compounds characteristic of sinus-related bacteria and infected sinus mucus: analysis by solid-phase microextraction and gas chromatography-mass spectrometry. J Chromatogr B Analyt Technol Biomed Life Sci 2009, 877:2011-2018.

31. Savelev SU, Perry JD, Bourke SJ, Jary H, Taylor R, Fisher AJ, Corris PA, Petrie M, De Soyza A: Volatile biomarkers of pseudomonas aeruginosa in cystic fibrosis and noncystic fibrosis bronchiectasis. Lett Appl Microbiol 2011, 52:610-613.

32. Lee TW, Brownlee KG, Conway SP, Denton M, Littlewood JM: Evaluation of a new definition for chronic pseudomonas aeruginosa infection in cystic fibrosis patients. J Cyst Fibros 2003, 2:29-34.
33. Vandendool H, Kratz PD: A generalization of the retention index system including linear temperature programmed gas-liquide partition chromatography. J Chromatogr 1963, 11:463-471.

34. Ooms K: Identification of potentially causal regressors in PLS models. Dissertation: International Study Program in Statistics. KUL; 1996.

35. Horvath I, Hunt J, Barnes PJ, et al: Exhaled breath condensate: methodological recommendations and unresolved questions. Eur Respir J 2005, 26:523-548.

36. Westhoff M, Litterst P, Freitag L, Urfer W, Bader S, Baumbach Jl: Ion mobility spectrometry for the detection of volatile organic compounds in exhaled breath of patients with lung cancer: results of a pilot study. Thorax 2009, 64:744-748

37. Buszewski B, Kesy M, Ligor T, Amann A: Human exhaled air analytics: biomarkers of disease. Biomed Chromatogr 2007, 21:553-566.

38. Vandendriessche T, Keulemans J, Geeraerd A, Nicolai BM, Hertog MLATM: Evaluation of fast volatile analysis for detection of botrytis cinerea infections in strawberry. Food Microbiol 2012, 32:406-14.

39. Wang T, Pysanenko A, Dryahina K, Spaněl P, Smith D: Analysis of breath, exhaled via the mouth and nose, and the air in the oral cavity. J Breath Res 2008, 2:037013.

40. Hansen SK, Rau MH, Johansen HK, Ciofu O, Jelsbak L, Yang L, Folkesson A, Jarmer HØ, Aanæs K, von Buchwald C, Høiby N, Molin S: Evolution and diversification of pseudomonas aeruginosa in the paranasal sinuses of cystic fibrosis children have implications for chronic lung infection. ISME J 2012, 6:31-45.

doi:10.1186/1465-9921-13-87

Cite this article as: Goeminne et al:: Detection of Pseudomonas aeruginosa in sputum headspace through volatile organic compound analysis. Respiratory Research 2012 13:87.

\section{Submit your next manuscript to BioMed Central and take full advantage of:}

- Convenient online submission

- Thorough peer review

- No space constraints or color figure charges

- Immediate publication on acceptance

- Inclusion in PubMed, CAS, Scopus and Google Scholar

- Research which is freely available for redistribution 